North and South America (see the cover). The new transition path from Lake Tecopa, for example, passes near this region.

The clustering of the transitional VGPs not only revives the old hypothesis that the transitional field is predominantly dipolar, but also requires the sequence of intermediate dipole orientations to be similar from reversal to reversal. This persistence is intriguing. It hints that slowly varying inhomogeneities in the temperature or con-

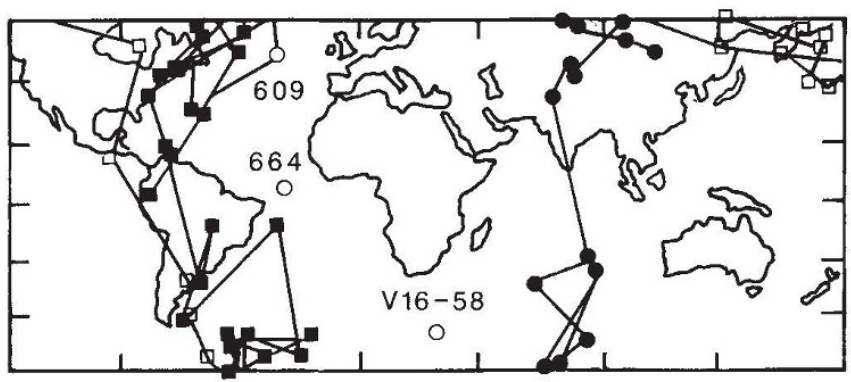

FIG. 2 Map after Clement and Kent ${ }^{9}$ showing antipodal VGP paths for the Matuyama-Brunhes reversal. Sampling sites are shown with open circles. The authors' conclusion: the transitional field was not dipolar and was not axially symmetric.

ductivity of the lower mantle are affecting the much more rapidly varying dynamo process in the outer core. As Laj and colleagues point out, it may be significant that both primarywave seismic velocity in the lower mantle and fluid flow in outer core are distinctive beneath the region traversed by so many transitional VGP paths.

The VGP paths also show a preference for the longitudinal band on the opposite side of the globe, between India and Australia. This observation certainly complicates the simple dipolar interpretation, and the Matuyama-Brunhes reversal illustrates how. Clement and $\mathrm{Kent}^{9}$ compare reversal records from the floors of the Atlantic and southwest Indian oceans at latitudes $49^{\circ} \mathrm{N}, 0^{\circ}$ and $46^{\circ} \mathrm{S}$. The transitional palaeomagnetic directions from the mid-latitude sites have the familiar dipolar look: the VGPs for both track through the Americas. As viewed from the Equator, however, this same transitional field produces a VGP track across India (Fig. 2). The dissimilar paths are not contradictory, but rather are evidence that the transitional field was strongly nondipolar. Clement ${ }^{10}$ demonstrates that an equatorial octupole field, for example, can produce antipodal VGP paths like those observed.

A second example is provided by Tric et $a l^{8}$. Their list includes seven records of the lower Jaramillo reversal, a reverseto-normal transition that preceded the Matuyama-Brunhes by several hundred thousand years. The data all come from midlatitude sites, three in the Southern Hemisphere and four from the Northern. Five of the sites show VGP paths centred over the Americas, but two, from either side of the Equator, show paths that lie about $90^{\circ}$ further west. Again, a nondipolar transitional field is much in evidence. Furthertion zones ${ }^{13}$. more, it is not like the transitional field of 200 thousand years later; VGP paths for the lower Jaramillo and Matuyama-Brunhes reversals from a single site in the North Atlantic are strikingly different ${ }^{11}$. This shortterm variability is not consistent with the idea that transitional fields are governed by lower-mantle structure.

Why is our new picture of the transitional geomagnetic field so different from previous ones? To some extent, the difference results from the many new data; the predominance of dipolar components during a reversal emerges only as a statistical tendency in this large collection. Could the pattern be fortuitous? Although 48 reversal records are included, only seven come from sites within 20 degrees of the Equator, and only five come from the Southern Hemisphere; geographical bias is still a worry. The scarcity of sedimentary transition zones with accumulation rates greater than $5 \mathrm{~cm}$ per thousand years is another difficulty. There is reason to question the fidelity of the palaeomagnetic recording process in slowly deposited sediments, especially in light of the evidence that intervals of short-lived, rapidly varying field may occur during the transitional stage ${ }^{12}$. It is even possible that persistent nondipole components, characteristic of the stable field, contaminate the remanence of such transi-

Nevertheless, it seems unlikely that the regularity identified by Laj and colleagues is entirely spurious. So does deep-mantle structure find expression in the transitional field? Perhaps. But as Laj and colleagues readily admit, it is far from clear how inhomogeneities of the lower mantle affect flow in the outer core, or how a particular flow pattern translates into systematic features of the transitional geomagnetic field.

Scott Bogue is in the Department of Geology, Occidental College, Los Angeles, California 90041, USA

\footnotetext{
1. Hillhouse, J. \& Cox, A. Earth planet. Sci. Lett. 29, 51-64 1976)

2. Laj, C. et al. Nature 451, 447 (1991).

3. Creer, K. \& Ispir, Y. Phys. Earth planet. Interiors 2 283-293 (1970).

4. Steinhauser, P. \& Vincenz, S. Earth planet. Sci. Lett. 19 113-119 (1973).

Valet, J.-P., Tauxe, L. \& Clark, D. Earth planet. Sci. Lett. 87 463-472(1988).

6. Hoffman, K. Science 196, 1329-1332 (1977).

7. Fuller, M., Williams, 1. \& Hoffman, K. Rev. Geophys, Space Phys. 17, 179-203 (1979)

8. Tric, E et al. Phys. Earth planet. Interiors 65, 319-336 (1991).

9. Clement, B. \& Kent, D. Geophys. Res. Lett. 18, 81-84 (1991)

10. Clement, B. Earth planet. Sci. Lett. (in the press)

11. Clement, B. \& Kent, D. Init. Rep. DSDP 94, 831-852 (1986).

2. Coe, R. \& Prevot, M. Earth planet. Sci. Lett. 98, 292-298 $1989)$

13. Rochette. P. Earth planet. Sci. Lett. 98. 33-39 (1990).
}

\section{Good resolutions}

Photographic film, says Daedalus, is a digital medium. A grain of silver halide in a bright region of the film has a high chance of being exposed and rendered developable; in a dark region, it has a low chance. After development, the resulting micromosaic of silver specks is a statistical digital sampling of the light-intensity distribution that hit the film.

This elegant chemistry has a singular flaw. An exposed grain becomes a speck of silver in the resulting negative, and you know where it is. An unexposed grain is dissolved by the fixer and you don't know where it was. In other words, the ' 1 's of the digital binary code are recorded but the ' 0 's are lost.

Daedalus now plans to remedy this defect. He recalls the fascinating physical chemistry of fossilization. As the organic remains of a dead organism slowly degrade and dissolve, their place is taken exactly by silica deposited from groundwater. So DREADCO chemists are inventing a photographic fixing solution supersaturated with silica. As it dissolves each unexposed grain of silver halide, it fills the resulting hole in the emulsion with a 'fossil' replica in white, light-stable silica. The negative thus records the ' 1 's of the optical sampling as black silver grains, and the ' 0 's as white silica grains. Even neater, the process can be inverted. The developed silver grains can be fossilized to white silica by a suitable solvent solution, and then the undeveloped silver halide grains are reduced to black silver. The result is a positive image with bright ' 1 's and dark ' 0 's, like the original scene. By elaborating the chemistry along established lines, with three layers of emulsion on the film each with its own colour filter, true positive three-colour photography should be possible.

'Fossilfilm, ${ }^{\text {क }}$ will look distinctly sharper and crisper than existing products. But only elaborate post-processing will reveal its full power. Its grain structure of ' 1 's and ' 0 's provides for the first time a true binary statistical sampling of the original optical field. From a set of microdensitometer scans, computer analysis will reconstruct a smoothed, best-fit two-dimensional optical image for each colour layer. Even lens aberrations could be removed, by deconvolution via the known transfer-function of the imaging lens. The results will be an ultra sharp, grainless, undistorted picture, containing all the information on the original film. Detail comparable to the grain separation distance should be recoverable.

Such post-processing will be expensive, of course. Most of the time it will not be needed. But every so often, when a photograph turns out to be a unique record of some dramatic event, or to contain crucial scientific or forensic evidence, Fossilfilm will come into its own.

David Jones 